\title{
Conducting polymer microactuators operating in air
}

\author{
Gursel Alici $^{1}$, Valerie Devaud ${ }^{2}$, Philippe Renaud ${ }^{2}$ and Geoff Spinks ${ }^{1}$ \\ ${ }^{1}$ University of Wollongong, School of Mechanical, Materials and Mechatronic Engineering, Australia \\ ${ }^{2}$ Swiss Federal Institute of Technology Lausanne (EPFL), Center of MicroNano Technology, Switzerland
}

Received 25 August 2008, in final form 28 November 2008

Published 20 January 2009

Online at stacks.iop.org/JMM/19/025017

\begin{abstract}
We report for the first time on microsized conducting polymer actuators, which operate in air and in liquids. These actuators are potentially useful for a wide range of applications from biotechnology to microrobots. Furthermore, the actuators are fabricated using an excimer laser ablation technique, which does not require clean-room facilities and can provide high throughput production. Preliminary characterization results presented show that the tip displacement of the microactuators is linearly proportional to the magnitude of the input voltage.
\end{abstract}

(Some figures in this article are in colour only in the electronic version)

\section{Introduction}

Electroactive polymers (EAPs) are considered a potential alternative to conventional actuators due to their attractive properties, including minimal electric power consumption, lightweight and compliant properties, biocompatibility, ability to operate in air and fluid, insensitivity to magnetic fields and simple fabrication. Furthermore, the scaling down of these EAPs (e.g. to the micron level) greatly improves their actuation properties such as the blocking force. As recognition of their attractive properties, a number of studies on conducting polymer actuators, including ionic and non-ionic systems, have been carried out, particularly on assessing their suitability for use at the macro down to the nanometre level. Small-sized EAP actuators are useful in numerous applications, including the micromanipulation of living cells, bioanalytical nanosystems, datastorage, lab on chip, microvalve, microswitch, microshutter, cantilever light modulators, micro-optical instrumentation, artificial muscles for macro/microrobotics and so on.

The area relevant to this paper is the microfabrication of conducting polymer actuators. In fact, very successful devices have previously been fabricated and demonstrated as useful in biotechnology to handle a single cell and similar $[3,6]$. Jager et al [11] fabricated a serially connected micromanipulator to pick, move and place $100 \mu \mathrm{m}$ glass beads. The micromanipulator is very suitable for single-cell manipulation. These systems, however, could only operate in specific aqueous media, as the media provided a source of ions (electrolyte) necessary to achieve actuation. Although significant attempts have been directed to the synthesis, performance quantification, positioning improvement and applications of the air-operating trilayer conducting polymer actuators based on polypyrrole (PPy), the systems studied to date had actuator dimensions greater than $2 \mathrm{~mm}$ [7-10]. There has not been any previously published work on microsized almost-solid state polymer actuators. The trilayer actuator design using a porous separator cannot be easily fabricated using lithography methods. With this in mind, we propose to use excimer laser ablation to fabricate conducting polymer microactuators. Actuators of various sizes with lengths ranging from 500 to $900 \mu \mathrm{m}$, widths between 160 and $350 \mu \mathrm{m}$ and thicknesses of $140-170 \mu \mathrm{m}$ were realized and tested. The main limiting factor here is the thickness of the actuator, which is approximately $170 \mu \mathrm{m}$. The smaller is the thickness, the more compact microactuators can be fabricated using the laser ablation technique with a high throughput. Our on-going research activity is directed at reducing the overall thickness. In the current structure, most of the thickness comes from a passive layer (poly(vinylidene fluoride) (PVDF)) which serves as a cell separator and 'the storage reservoir' of the salt and the solvent. Using a solid electrolyte with a thickness of $20 \mu \mathrm{m}$ will allow us to make microactuators, for example, with the dimensions of $200 \mu \mathrm{m} \times 50 \mu \mathrm{m} \times 50 \mu \mathrm{m}$ and even smaller. Even with the actuators with the maximum dimension of approximately $900 \mu \mathrm{m}$, this is a significant step in realizing all-solid state microdevices articulated with novel materials such as electroactive polymers. 


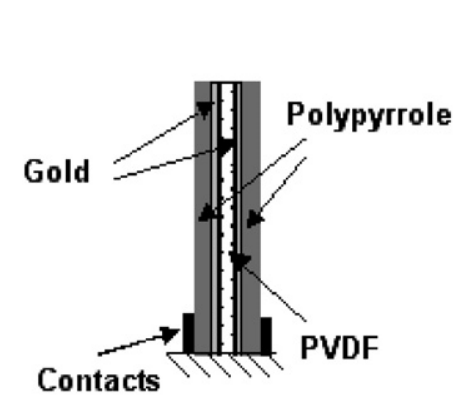

(a)

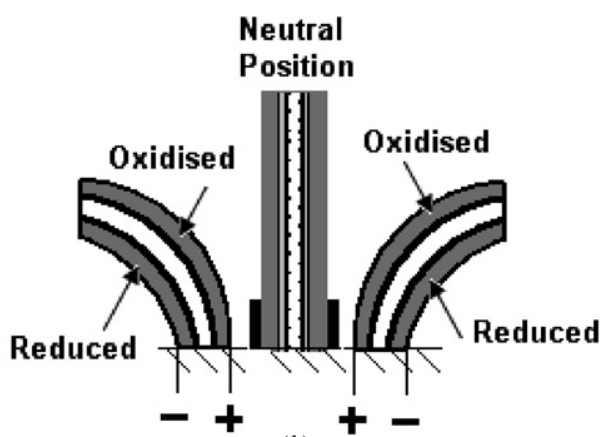

(b)

Figure 1. (a) Schematic structure of the PPy trilayer actuator and $(b)$ schematic representation of the bending principle. This actuator is anion-driven; the anions of the electrolyte which is held inside the pores of the PVDF layer move to the positively charged electrode as the PPy is oxidized to cause a volume expansion. At the negative electrode, the PPy is reduced causing expulsion of anions and volume shrinkage.

Actuator materials currently used for micro-electromechanical systems (MEMS) suffer several limitations. The ability to mechanically grasp and hold an object (e.g. gripping), for example, is difficult using conventional MEMS actuators (e.g. electrostatic systems). Furthermore, actuators made from inorganic materials face two key problems: (1) the implementation of large design footprints or areas and (2) the brittleness of the material. Conventional actuators suffer from relatively large footprints that limit the degree of miniaturization and actuator density. In contrast, polymer thermal actuators [4] have been fabricated into arrays to achieve high actuator densities and as such their parallel arrangements can be exploited for high power applications such as the handling of large objects. The brittleness of inorganic materials means that they readily break upon contact with macro-scale objects. Polysilicon microgrippers have been known to break if touched by a macro-object or if exposed to air velocities higher than $1 \mathrm{~m} \mathrm{~s}^{-1}$ [5]. In contrast, the compliant properties of the polymer actuators allow the resumption of normal operation after the actuator has been subject to significant mechanical forces or disturbance [6]. By incorporating electroactive polymers in MEMS, the fabrication of the actuator elements is simplified. Simple EAP coatings can replace complex interdigitated comb drives, multi-layer piezo systems and bring new functionality not available in silicon-based systems. For MEMS devices, the advantages of EAPs include their larger actuation at lower voltages, operation in air and wet media, biocompatibility and additional functionality for controlled drug release and chemical sensing applications.

In this paper, we follow a hybrid microfabrication technique, consisting of the bottom-up approach of making a composite structure including the two electroactive polymer layers and the top-down approach of the excimer laser ablation technique, which is applied to produce the microactuators. The actuators are a microscopic version of the previously reported polypyrrole 'trilayer' structure that consists of two PPy layers sandwiched on either side of a porous separator that contains an electrolyte. Such macrosized trilayers operate in the bending mode, and fast operation has been previously reported.

\section{Synthesis and operation principle of actuators}

Figure 1 illustrates the structure of the bending-type polymer actuators reported in this paper. There are three main layers in the structure: two outer PPy layers that are active components and an inner porous separator of poly(vinylidene fluoride) (PVDF) that holds the liquid electrolyte. The 'trilayer' structure also includes two thin, porous gold layers as shown in figure 1. This laminated structure behaves like a bilayer, generating a simple bending motion. The active polymer layers generate a volume change during electrochemical oxidation and reduction when a voltage is applied. However, with the passive PVDF layer in permanent contact with the PPy layers the composite actuator bends when it is cantilevered, as shown in figure 1.

The fabrication of the trilayer structure starts with the sputter coating of gold particles (the thickness ranging between 10 and $100 \AA$ ) on both sides of a PVDF sheet. PDVF is a commercially available filter membrane with a pore size of $0.45 \mu \mathrm{m}$ and a nominal thickness of $110 \mu \mathrm{m}$ (Millipore). The coated layers of gold serve to provide a conductive surface on which the PPy electrodes can be electrochemically deposited. Propylene carbonate (PC, Aldrich) and lithium triflouromethanesulfonimide $\left(\mathrm{Li}^{+} \mathrm{TFSI}^{-}\right.$, $3 \mathrm{M})$ were used as received. Pyrrole (Merck) was distilled and stored under nitrogen at $-20{ }^{\circ} \mathrm{C}$ before use. Using a potentiostat/galvanostat, the polypyrrole layers were grown galvanostatically on the gold-coated PVDF at a current density of $0.1 \mathrm{~mA} \mathrm{~cm} \mathrm{~cm}^{-2}$ for $12 \mathrm{~h}$ from the growth solution. The solution contains $0.1 \mathrm{M}$ LiTFSI, $0.1 \mathrm{M}$ pyrrole monomer and $1 \%$ water in PC, stirred and degassed with $\mathrm{N}_{2}$ for $15 \mathrm{~min}$. With this growing time, the thickness of the polymer layers was approximately $30 \mu \mathrm{m}$, making the overall thickness $170 \mu \mathrm{m}$. The deposition temperature was $-33^{\circ} \mathrm{C}$. The synthesized PPy is doped with the $\mathrm{TFSI}^{-}$ion during polymerization. Upon completion of the polymerization, the polymer (PPy) coated bulk sheet was rinsed with acetone to remove any remaining growth solution and stored in the electrolyte solution (without a pyrrole monomer) until it was needed.

With the electrolyte stored in the cell separator, the trilayer structure forms an electrochemical cell. When a potential difference or current is passed between the polymer (PPy) 


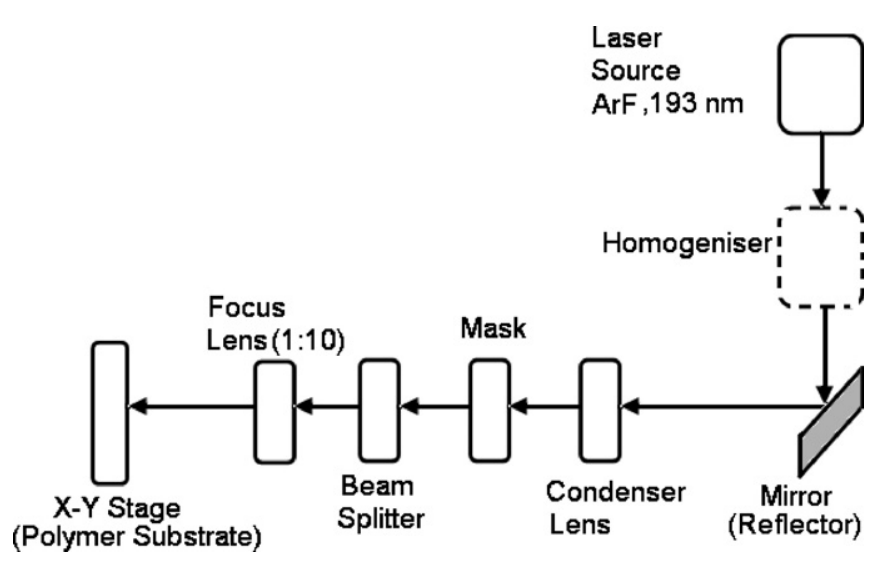

Figure 2. Configuration of the excimer laser ablation system (not to scale).

electrodes via the contacts, the whole structure is charged like a battery: at the positively charged electrode the PPy layer is oxidized, while the negatively charged PPy is reduced. To maintain charge neutrality within the PPy layers, TFSI ${ }^{-}$ anions will move from the electrolyte to the positively charged polymer (PPy) electrode and hence cause a volume expansion. While this is happening in the positive electrode/anode, the anions $\left(\mathrm{TFSI}^{-}\right.$) will leave the negatively charged electrode as reduction of the PPy causes it to become uncharged and a volume contraction occurs. The overall result is that the cantilevered structure will bend towards the negative electrode/cathode, as depicted in figure $1(b)$. The volume change happens due to movement of the charge balancing anions in and out of the polymer layers, and perhaps some solvent molecules move inside the polymer layers, due to osmotic effects, to balance the ionic concentration. The charge transfer between the anode and cathode determines the volume change. Electrostatic forces between the displaced ions and the polymer backbone are also believed to contribute to the bending [7]. The actuation speed and the overall volume change depend on many electrochemomechanical parameters including the thickness of the polymer layers, the ion type and sizes, charge injected (potential applied), the ionic concentration, the solvent and the width of the actuator $[10,12]$.

\section{Experimental set-up}

The schematic representation of the excimer (excited dimer) laser ablation system used in this study is shown in figures 2 and 3. The excimer laser (LPX305 Lambda Physik), which is an ArF laser, is based on high-pressure plasma consisting of the noble gas argon and reactive gas fluorine $(F)$ with a wavelength of $193 \mathrm{~nm}$, pulse duration of $2 \mathrm{~ns}$, maximum power of $25 \mathrm{~W}$ and pulse rate of $50 \mathrm{~Hz}$. From the mask, the laser beam passes through a beam splitter and is then projected onto the sample through a focus lens with a demagnification factor of 1:10. The final beam will generate a uniformly exposed area of $1 \times$ $1 \mathrm{~mm}^{2}$ on the motorized $X-Y$ stage, which has a supplierspecified resolution of 1 micrometre. But, when we made the microactuators, the stage was in the static mode. The other fabrication conditions are 2000 pulses at $50 \mathrm{~Hz}$ and $300 \mathrm{~mJ}$

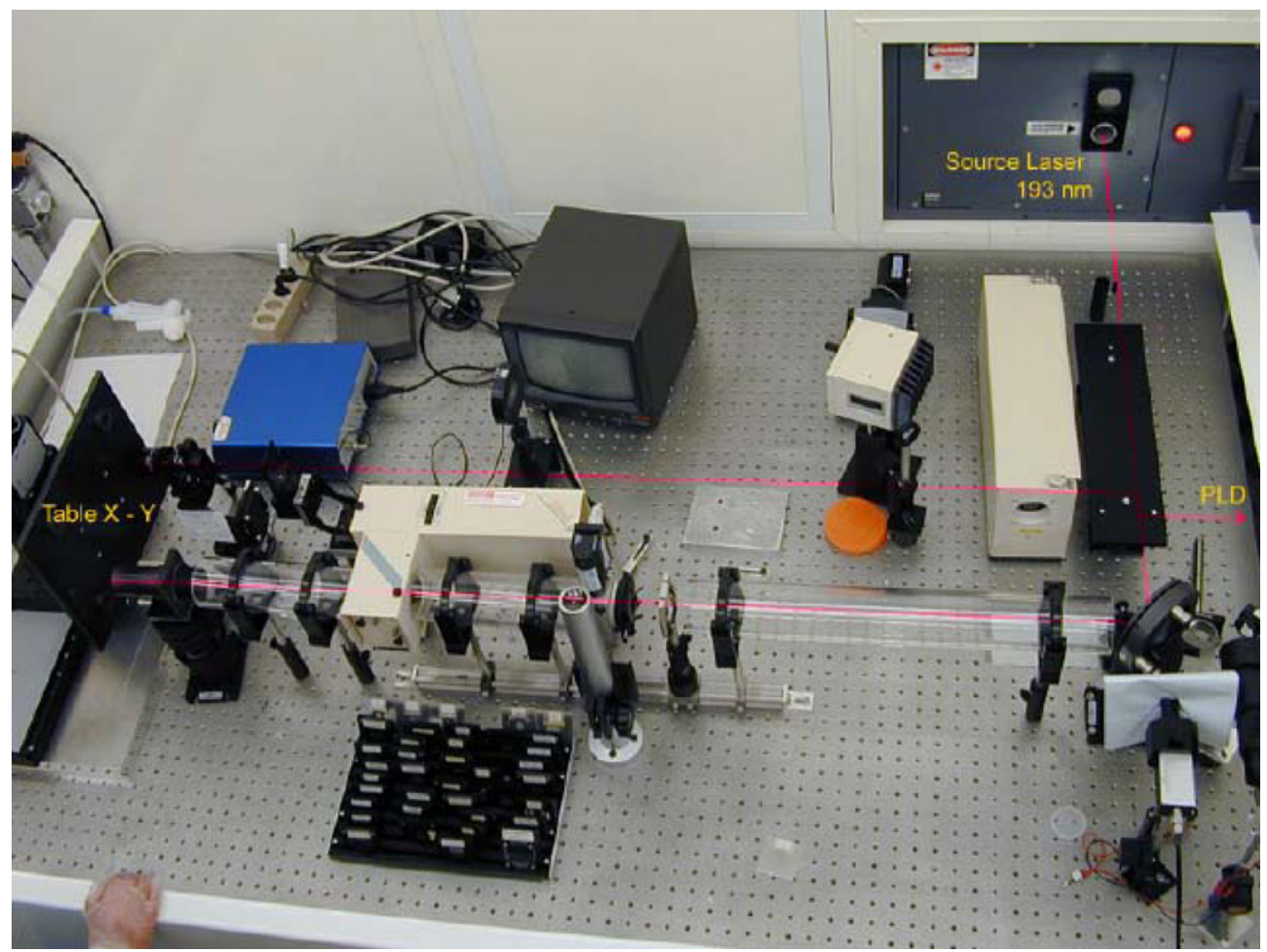

Figure 3. A photographic view of the excimer laser ablation system used. 


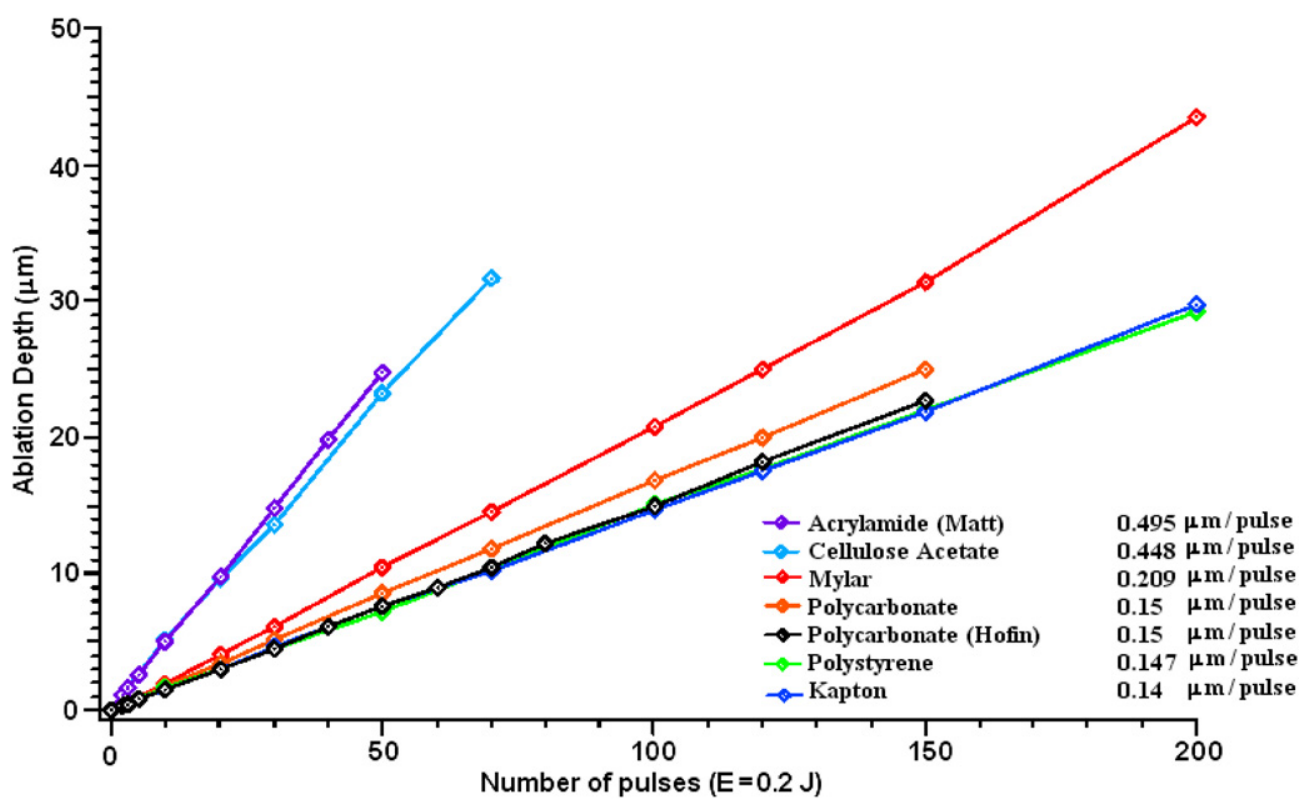

Figure 4. Variation of the ablation depth with the pulse number for various materials. These data are for the excimer laser employed in this study [23].

of energy at the source. The fluence of the final beam was approximately $9 \mathrm{~mJ} \mathrm{~mm}^{-2}$.

Excimer lasers are based on a mixture of gases providing beams in the UV bandwidth with short pulse rates and durations, which supply high energy intensity $\left(\mathrm{J} \mathrm{cm}^{-2}\right)$. The higher are the energy intensity and the number of pulses, the higher is the depth of cut or ablation. From the fabrication point of view, advantages of excimer lasers include their ability to directly machine a substrate and the fact that they do not require clean-room facilities. If the beam is shaped and demagnified properly, the laser can make parallel cutting/ablation of multiple shapes, i.e. high throughput. Although an excimer laser beam cannot be focused on a small area, the laser beam can be demagnified to fabricate multiple microstructures through projection printing. Compared to direct writing techniques based on the solid-state lasers, the excimer laser ablation provides a high throughput. In other words, it is very suitable to mass fabrication of microstructures. Further, it can be used to machine or cut a range of materials including metals, glass, biomaterials, semiconductors, crystals, ceramic, polymers, piezomaterials, plastics and similar to make microstructures with the minimum feature of $1 \mu \mathrm{m}[1,2]$.

Ablation can happen in two different processes: photothermal and photochemical. While the first is the primary ablation mechanism when ablating metals, ceramic and glass, the second is for the ablation of polymers. As the structure of the trilayer polymer bender is mostly polymeric (with a negligibly small gold layer), we suggest that the ablation of the polymer trilayer structures is due to a photochemical process where the ablation occurs by directly breaking the atomic bonds of the composite structure without causing any thermal defects. This is an advantage in the sense that the cut regions will not be affected by any heating effect and creating structures with finer resolution compared to the same structures ablated with the photothermal process. It has been reported [2] that the excimer laser ablation is particularly suitable to operate on materials with low melting temperature and low thermal conductivity. These two requirements, too, are satisfied by our conducting polymer actuator.

The variation of the ablation rate with the pulse number for various materials is depicted in figure 4 . The ablation rates $(d)$ are also calculated for each material for completeness of these data. The fluence $\left(F_{\mathrm{i}}\right)$ which was kept at $1.85 \mathrm{~J} \mathrm{~cm}^{-2}$ must be above the threshold $\left(F_{\mathrm{t}}\right)$ for materials to be removed, rather than sending a large number of sub-threshold fluence pulses [1]. This fact is described by the Beer-Lambert principle:

$$
d=\frac{1}{\alpha} \operatorname{In}\left(\frac{F_{\mathrm{i}}}{F_{\mathrm{t}}}\right), \quad \text { for } \quad F_{\mathrm{i}}>F_{\mathrm{t}},
$$

where $\alpha$ is the effective absorption coefficient, which is determined experimentally from fluence versus ablation rate data.

\section{Fabrication of microactuators}

Although the excimer laser ablation technique has been applied to make various shapes and materials [1], it has not been applied to make microsized conducting polymer actuators. The microactuator in this paper is a microcantilever where the length/width and width/thickness ratios are at least 2.0. With this in mind, a set of actuators with the dimensions of $\sim(L=$ 500-900) $\mu \mathrm{m} \times(W=160-350) \mu \mathrm{m} \times(t=140-170) \mu \mathrm{m}$ were fabricated. As a first step, a UV-resistant molybdenum mask with a $1 \mathrm{~mm} \times 1 \mathrm{~cm}$ slit in it was prepared by using direct laser cutting (Nd:YAG laser). The polymer sheet was placed on the motorized $X-Y$ stage in front of the incoming laser beam passing through the mask. The stage was in the static mode when generating each of the three ablation lines in figure 5; the stage was moved to the next line position consecutively. 


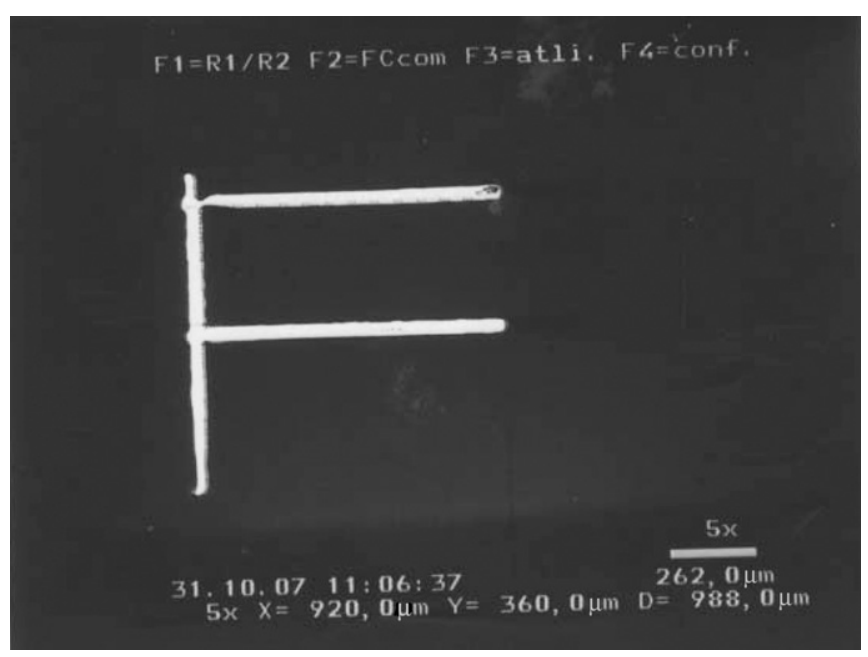

Figure 5. Micrograph of the laser ablation-fabricated microcantilever. It must be noted that the ablation lines (in white colour) are quite accurate, with no thickness change. This suggests that the laser beam energy is efficiently and properly conveyed to the ablation lines. The length and width of the $U$ shape between the ablation lines are $920 \mu \mathrm{m}$ and $360 \mu \mathrm{m}$, respectively.

The micrograph of one of the fabricated actuators is depicted in figure 5. The microcantilever was cut out and tested using a power supply set at $1 \mathrm{~V}$. The snapshots of two such actuators activated under $1 \mathrm{~V}$ are provided in figure 6.

\section{Characterization of microactuators}

Step response experiments were conducted to quantify the bending displacements. Based on the bending displacement, the induced bending moment (the driving force of the actuators) was calculated. For the step response experiments, a single-axis laser displacement sensor was used to measure the horizontal deflection of the cantilevered microactuator. A custom-built experimental system was used to apply an arbitrary voltage input to the trilayer bender actuator and measure its response (figure 7) [22]. The actuator input voltage signal was provided by an external function generator and amplified using an eDAQ potentiostat (eDAQ, model EA161) operating in a two-electrode mode. A non-contact laser displacement sensor (micro-epsilon, model NCDT-170010) was used to measure the displacement of the actuator tip, outputting a proportional voltage signal. The resolution of the laser displacement sensor is high, at $0.5 \mu \mathrm{m}$, but the measurement electronics introduces a $2 \mathrm{~ms}$ delay. A datalogger (e-corder, model ED821) recorded the voltage signal applied to the trilayer, the associated current drawn and the displacement voltage signal from the laser displacement sensor. The e-corder is an interface unit between the computer, the laser displacement sensor and the potentiostat.

The input voltage was changed from $0.2 \mathrm{~V}$ to $3 \mathrm{~V}$ peakto-peak square waves. The steady state values of the actuator response under these input voltages were measured and are
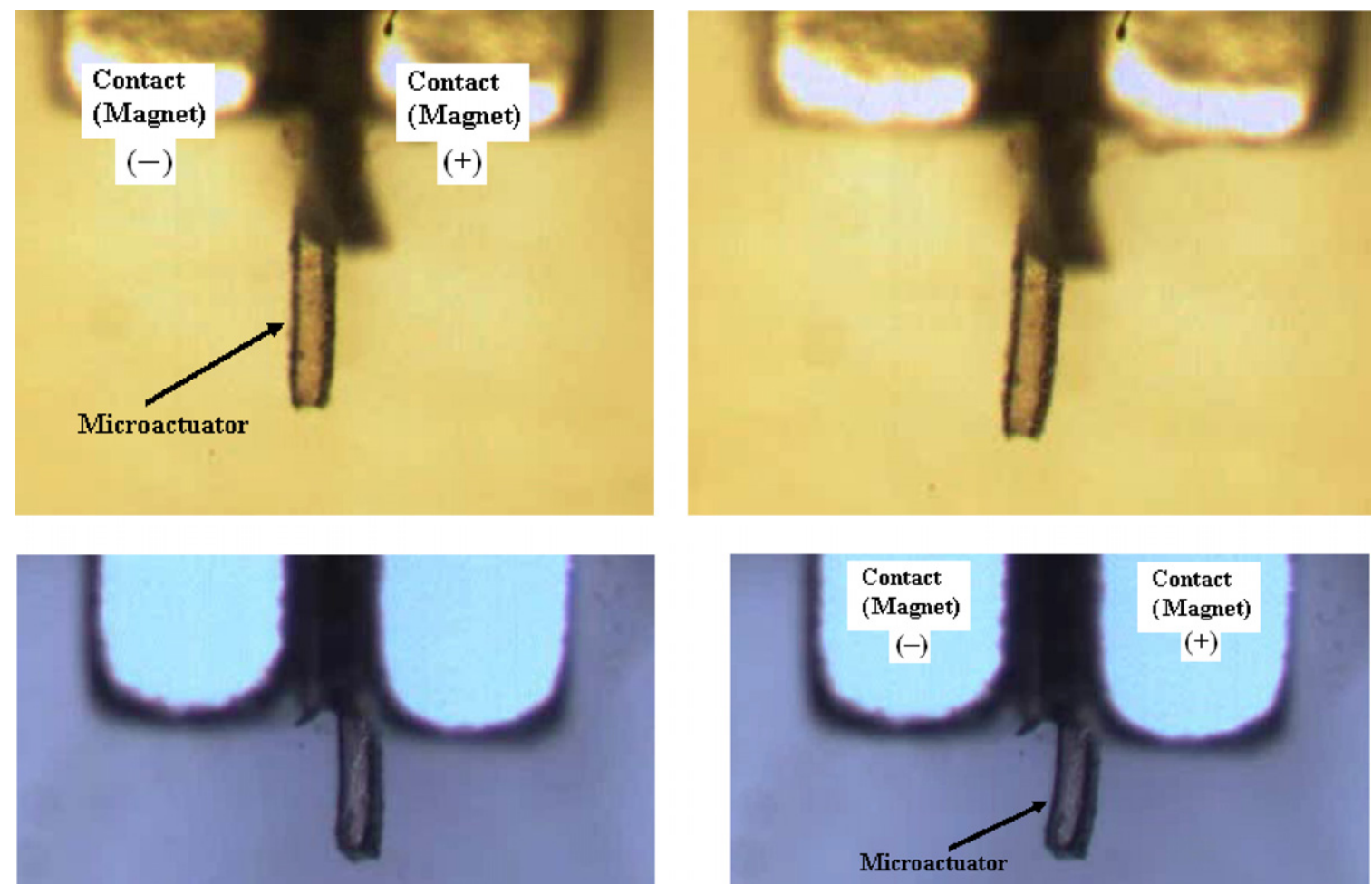

Figure 6. Photographs of the side views of two microactuators under $1 \mathrm{~V}$. The images on the left show the neutral positions of the actuators. Two permanent magnets are used to create enough compaction force on two electrically conducting metal plates, between which the microactuator is placed. Photographs on the right show bending displacement towards the negative electrode (cathode). The sizes of the top and bottom actuators are $920 \mu \mathrm{m} \times 360 \mu \mathrm{m} \times 170 \mu \mathrm{m}$ and $850 \mu \mathrm{m} \times 250 \mu \mathrm{m} \times 170 \mu \mathrm{m}$, respectively. 


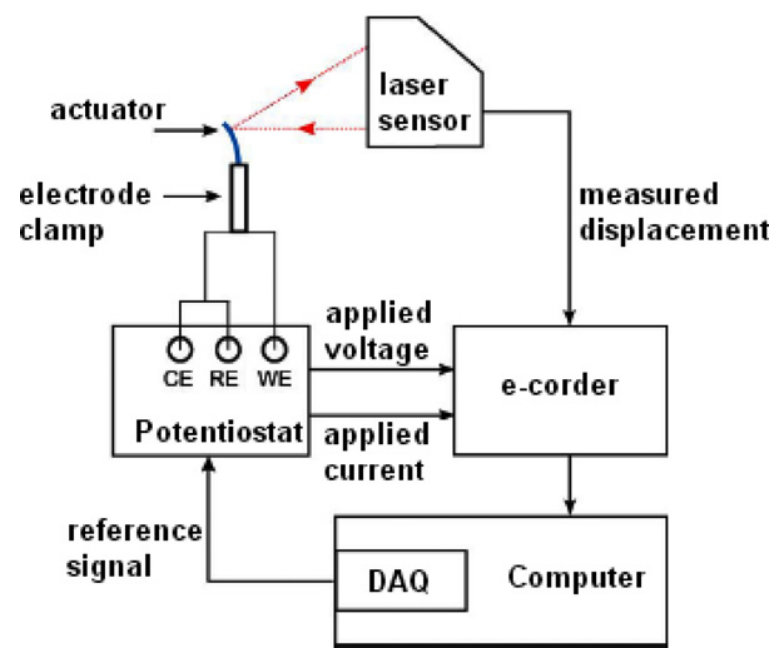

Figure 7. Schematic of the experimental set-up, where the laser sensor is mounted on an $X-Y$ micrometre stage. CE, RE, WE are the counter electrode, the reference electrode and the working electrode, respectively.

provided in figure 8 , which indicates that the tip displacement is proportional to the input voltage. This finding is in agreement with our previous studies of trilayer macroactuators $[7,21]$. Further, it was noted that the displacement of the microactuator tip under $2 \mathrm{~V}$ peak to peak or $1 \mathrm{~V}$ step voltage input is approximately half of the length of the actuator. This finding is also in agreement with our previous investigations [7]. A typical step response under a $2 \mathrm{~V}$ peak-to-peak square wave is depicted in figure 9. The micrograph of this actuator is provided in figure 10 .

The tip displacement $x$ of the microactuator measured by the laser distance sensor can be related to the radius of the curvature $r$ via a simple geometric approach [21]:

$$
\frac{1}{r}=\frac{2 x}{x^{2}+q^{2}}
$$

where $q$ is the distance between the clamped end and the laser incident point when the beam is at rest, as shown in figure 11. Equation (2) is obtained from $x^{2}+q^{2}$, where $q=r \sin \theta$ and $x=r(1-\cos \theta)$. For small bending $(x<q)$, the curvature is approximately linear with respect to the displacement:

$$
\frac{1}{r}=\frac{2 x}{q^{2}} .
$$

With reference to figure 8 , this relationship also suggests that the reciprocal of the bending curvature is also linearly proportional to the input voltage.

Bernouilli-Euler suggest that the bending moment on a loaded beam is proportional to the change in the curvature along the actuator length $L$ [24]. Using the parameters described in figure 12, this principle is governed by the following expression. The radius of curvature of a cantilever beam, i.e. the microactuators, is given by

$$
\frac{1}{r}=\frac{M_{\text {internal }}}{E I}=\frac{\mathrm{d} \theta}{\mathrm{d} L},
$$

where $\theta$ is the slope of the deflected beam. From equations (2) and (4), the bending moment induced upon the microcantilever

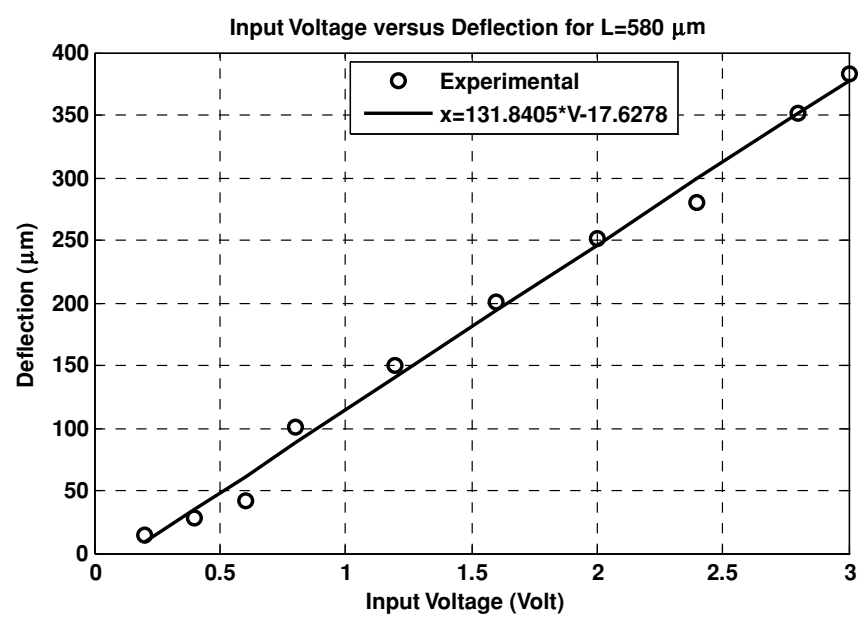

Figure 8. Variation of the tip displacement of the microactuator with step voltages ranging from $0.2 \mathrm{~V}$ to $3 \mathrm{~V}$ peak to peak for the microactuator with the dimensions of $580 \mu \mathrm{m} \times 220 \mu \mathrm{m} \times$ $160 \mu \mathrm{m}$. A first-order polynomial fitted to these experimental in a least square manner must be noted. The curve fit clearly shows that the actuator tip displacement is linearly proportional to the input voltage over the range of voltages studied.

as a result of the electrochemomechanical actuation is

$$
M_{\text {internal }}=\frac{2 x}{q^{2}+x^{2}} E I \text {. }
$$

Knowing the flexural rigidity $E I$ and the radius of curvature $r$ through experimentation will allow us to calculate the induced bending moment causing this deflection $x$. With reference to figure 13 , the flexural rigidity is

$$
E I=2 b\left(\frac{h_{1}^{3}}{3} E_{\mathrm{PVDF}}+\frac{h_{2}^{3}-h_{1}^{3}}{3} E_{\mathrm{PPy}}\right) \text {, }
$$

where $E_{\mathrm{PPy}}$ and $E_{\mathrm{PVDF}}$ are the elastic moduli of the PPy and PVDF, respectively. For the actuators used in this study, $E_{\mathrm{PPy}}$ and $E_{\mathrm{PVDF}}$ are estimated using the resonance frequency of the actuators identified through frequency response experiments [22] as $120 \mathrm{MPa}$ and $127 \mathrm{MPa}$, respectively. For the actuator dimensions of $L=580 \mu \mathrm{m}, b=220 \mu \mathrm{m}$ and $t=160 \mu \mathrm{m}, E I$ is calculated to be $8.9429 \times 10^{-9} \mathrm{~N} \mathrm{~m}^{2}$. Using equation (5) for $q=560 \mu \mathrm{m}$, the internal bending moment is calculated for the tip displacement depicted in figure 8 , and is presented in figure 14 .

\section{Applications}

The applications of such all-solid state microactuators with a small footprint and compliance are numerous, especially in the MEMS domain. The active microcantilevers can be used as the lids of microvials to keep small amounts of a chemical and prevent evaporation or leakage, and to keep a single cell or single cell organisms for biotechnology applications, especially the mobile and non-adhesive ones [14-16]. It can be used as a cell tapper, too, to investigate 'the effect of mechanical stimulation on a single cell [3]'. Another application can be in the microfluidics area to use them as valves to adjust flow in microchannels [17]. Similarly, the microactuators can be used as valves in the 

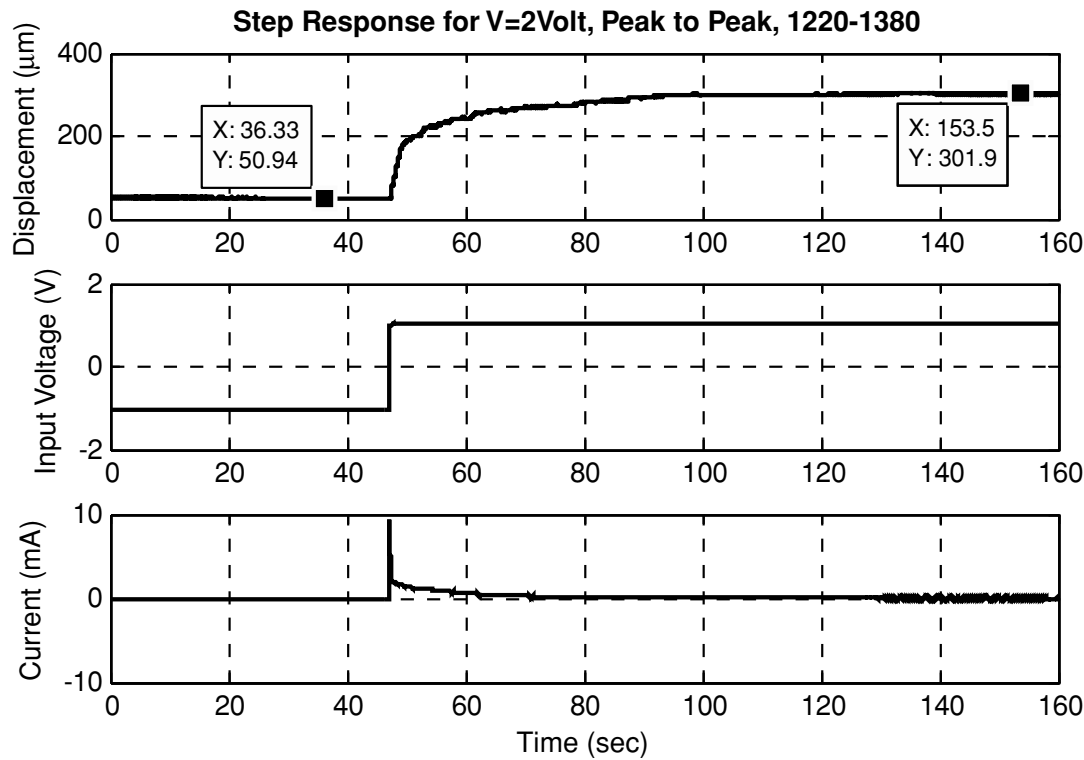

Figure 9. The tip deflection and current of the microactuator with the dimensions of $580 \mu \mathrm{m} \times 220 \mu \mathrm{m} \times 160 \mu \mathrm{m}$ under a step input from $-1 \mathrm{~V}$ to $+1 \mathrm{~V}$. It must be noted that mechanical time constant (from the top plot) is much larger than the electrical time constant (from the bottom plot). This follows that the mechanical dynamics dominate the response of the microactuators.

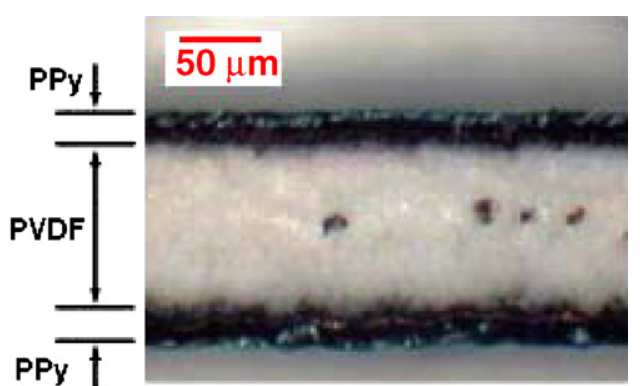

Figure 10. Micrograph of the microactuator with the dimensions of $580 \mu \mathrm{m} \times 220 \mu \mathrm{m} \times 160 \mu \mathrm{m}$. The thickness of the PVDF layer (white section) is $110 \mu \mathrm{m}$, and those of the black layers (predominantly PPy) are $25 \mu \mathrm{m}$, which was grown in $12 \mathrm{~h}$. There are thin layers of sputter-coated gold between the PPy layer and the PVDF layer.

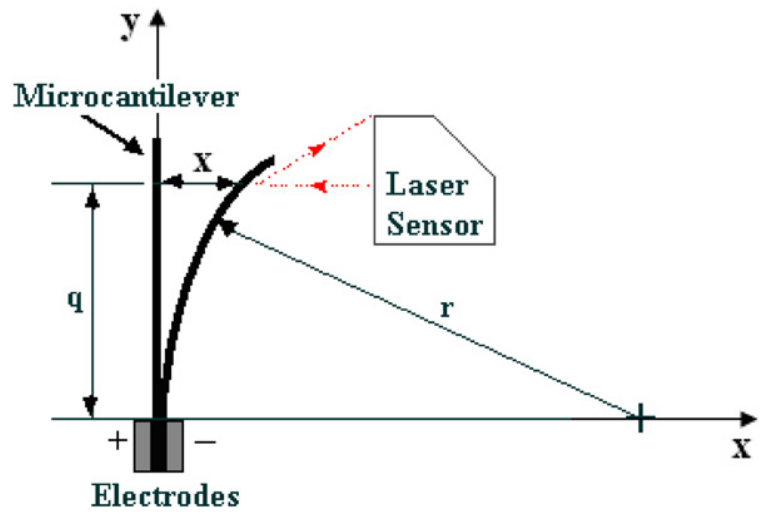

Figure 11. Configuration of the laser displacement sensor and the measurement parameters describing the radius of the curvature.

area of drug delivery to release drug. Of course, the most popular application would be in microrobotic applications, where the challenge is to establish wireless and autonomous microrobots [18, 19]. As the fabricated microactuators

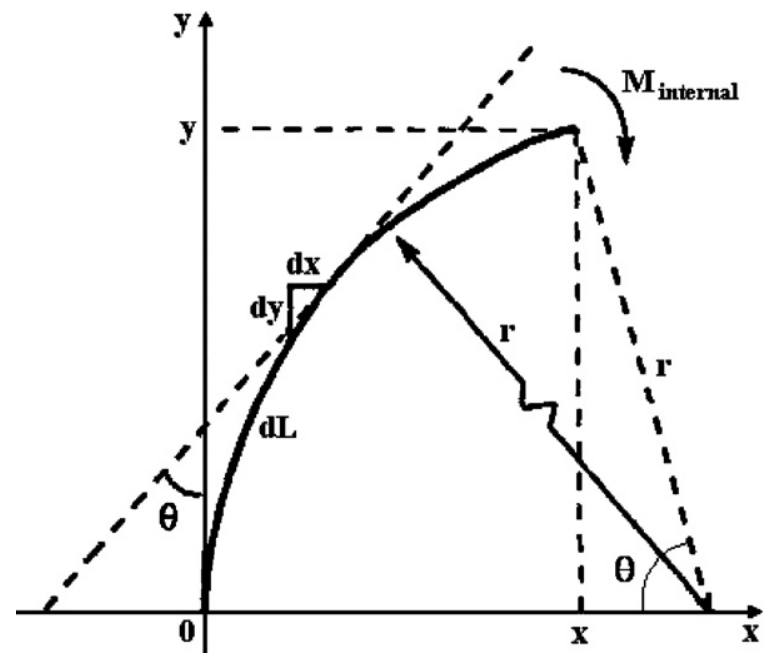

Figure 12. The parameters describing the relationship between the radius of the curvature and the bending moment causing the tip displacement of the cantilever beam (microactuator).

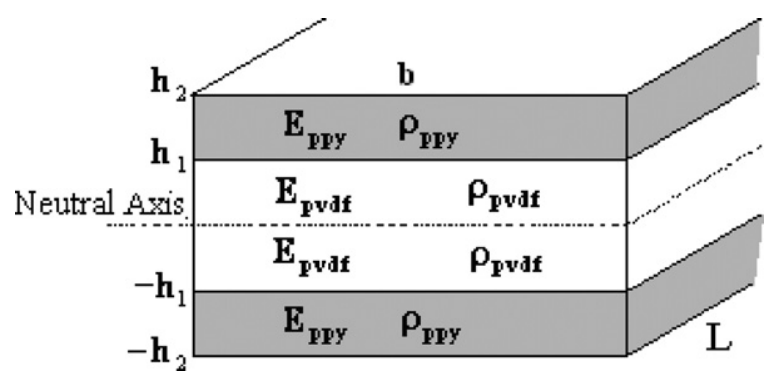

Figure 13. Description of the geometric parameters to formulate the flexural rigidity of the trilayer polymer actuators.

consume a very low electric power, and more importantly have a small footprint (no sophisticated electronics or additional gears or other mechanisms), they are especially suitable to 


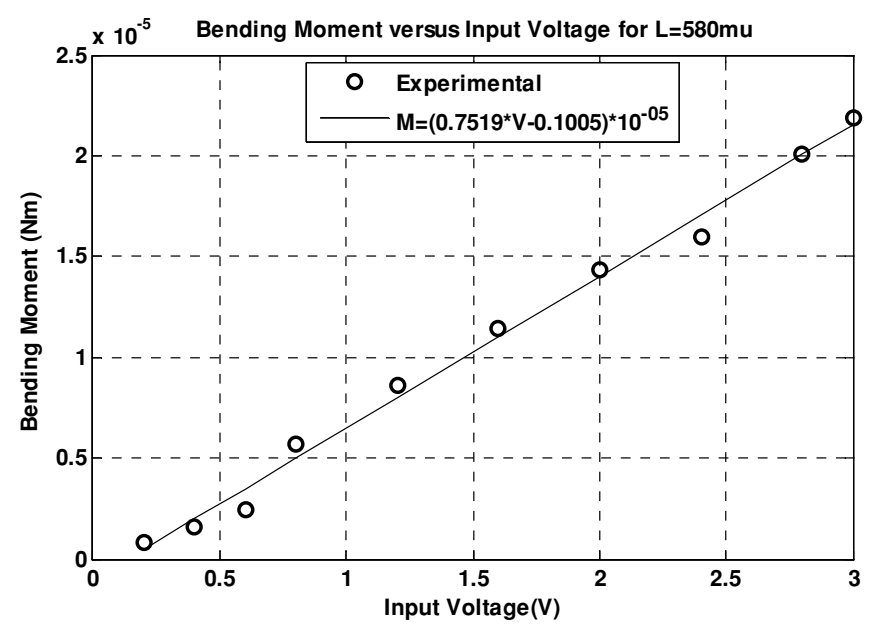

Figure 14. Variation of the induced internal bending moment of the microactuator with step voltages ranging from $0.2 \mathrm{~V}$ to $3 \mathrm{~V}$ peak to peak for the microactuator with the dimensions of $580 \mu \mathrm{m} \times$ $220 \mu \mathrm{m} \times 160 \mu \mathrm{m}$. A first-order polynomial fitted to these experimental in a least square manner must be noted. This curve fit clearly shows that, like the tip displacement, the induced bending moment is linearly proportional to the input voltage. This is in agreement with our previous study on macroactuators [12].

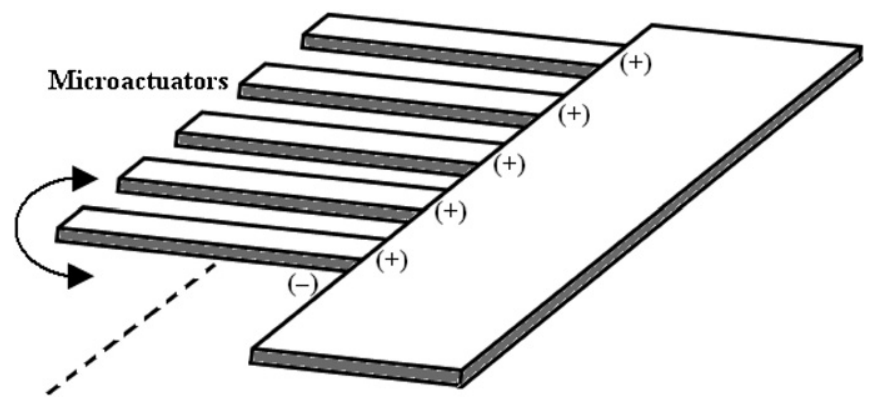

Figure 15. Schematic representation of microactuator arrays.

articulating microsized devices such as microrobots. It must be noted that any microactuator with remote power supply will be constrained by wirings to prevent functional movement. Using excimer laser ablation, arrays of the microactuators, as illustrated in figure 15, can be easily fabricated. The multiple actuators can be powered simultaneously through a single onsite power supply to realize any micro/nano manipulation tasks with high force outputs. Two of such microcantilevers can be assembled in parallel to form a two-finger robotic microgripper [7].

Another application is to employ the microcantilever as a micromirror holder (modulating element) in microoptoelectromechanical systems (MOEMS). The micromirrors are essential components of microscopes, scanners, optical switches, projection displays, interferometers and similar. Traditionally, microstructrues holding the mirrors are fabricated using surface and bulk micromachining techniques from polysilicon, metals and similar materials, but require high operation voltages ranging from $18 \mathrm{~V}$ to $75 \mathrm{~V}$ [20]. It must be recalled that the microcantilever based on the conducting polymer actuator requires typically $1 \mathrm{~V}$ and $15 \mathrm{~mA}$. A typical configuration of a micromirror is illustrated in figure 16.

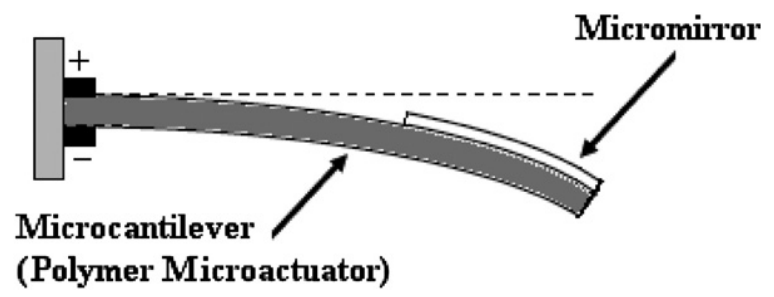

Figure 16. Schematic representation of a micromirror oriented through the polymer microcantilever, which serves as a light modulator.

\section{Conclusions and future work}

Using the excimer laser ablation technique, we have fabricated conducting polymer microactuators, capable of operating in air. To the best of our knowledge, this is the first attempt to make microsized polymer actuators by using the excimer laser ablation technique. More importantly, it is the first time that conducting polymer microactuators have been operated in air. All previous conducting polymer actuators could only be operated when immersed in a liquid electrolyte. The applications of such actuators are boundless, ranging from biotechnology to microrobotics, hence making another step in the development of microsized, small footprint and compliant actuators. With the realization of such actuators, it is the time to establish microsized smart devices (MEMS and MOEMS) based on EAPs.

Future work includes further miniaturization of the actuators, pushing the limits towards nanosized solidstate devices, and a comprehensive characterization and performance quantification of the microactuators including nonlinear effects, such as hysteresis and creep, demonstrate that the laser excimer technique is a high-yield fabrication technique, paving the way towards commercially viable applications, for example lab on chips.

\section{Acknowledgments}

The authors thank Dr Scott McGovern and Dr Stephen W John from the University of Wollongong for synthesizing the bulk sheet of the polymer actuators. Dr Stephen W John is acknowledged for providing figure 10. Furthermore, G Alici would like to thank the staff and research students of Robotics Systems Laboratories (LSR1 and LSR2) at Swiss Federal Institute of Technology Lausanne (EPFL) for their assistance and encouragement during the course of this study, and during his whole study leave. This work was supported in part by a URC Small Grant (2007) and ARC Discovery Project (DP0878931).

\section{References}

[1] Dyer P E 2003 Excimer laser polymer ablation: twenty years on Appl. Phys. A 77 167-73

[2] Tseng A A, Chen Y T, Chao C L, Ma K J and Chen T P 2007 Recent developments on microablation of glass materials using excimer lasers Opt. Lasers Eng. 45 975-92

[3] Jager E W H, Smela E and Inganas O 2000 Microfabricating conjugated polymer actuators Science 290 1540-5 
[4] Ataka M A, Omodaka A, Takeshima N and Fujita H 1993 Fabrication and operation of polyimide bimorph actuators for a ciliary motion system J. Microelectromech. Syst. 2146

[5] Kim D H et al 2005 A superelastic alloy microgripper with embedded electromagnetic actuators and piezoelectric force sensors: a numerical and experimental study Smart Mater. Struct. 14 1265-72

[6] Smela E 1999 Microfabrication of PPy microactuators and other conjugated polymer devices J. Micromech. Microeng. $91-18$

[7] Alici G and Huynh N N 2007 Performance quantification of conducting polymer actuators for real applications: a microgripping system IEEE/ASME Trans. Mechatronics $1273-84$

[8] Alici G, Spinks G M, Huynh N N, Sarmadi L and Minato R 2007 Establishment of a biomimetic device based on tri-layer polymer actuators-propulsion fins J. Bioinspir. Biomim. 2 S18-30

[9] Yao Q, Alici G and Spinks G M 2008 Feedback control of tri-layer polymer actuators to improve their positioning ability and speed of response Sensors Actuators A 144 176-84

[10] Wu Y, Alici G, Spinks G M and Wallace G G 2006 Fast tri-layer polypyrrole bending actuators for high speed applications Synth. Met. 156 1017-22

[11] Jager E W H, Inganas O and Lunstrom I 2000 Microrobots for micrometer-size objects in aqueous media: potential tools for single cell manipulation Science 288 2335-8

[12] Alici G, Metz P and Spinks G M 2006 A methodology towards geometry optimisation of high performance polypyrrole (PPy) actuators J. Smart Mater. Struct. $15243-52$

[13] Minato R, Alici G, McGovern S T and Spinks G M 2007 Tri-layer polymer actuators with variable dimensions Proc. SPIE 14th Int. Symp. Smart Structures and Materials, and Nondestructive Evaluation and Health Monitoring (San Diego, CA) vol 6524-57
[14] Jager E W H, Immerstrand C, Peterson K H, Magnusson K E, Lundstrom I and Inganas O 2002 The cell clinic: closable microvials for single cell studies Biomed. Microdevices 4 177-87

[15] Urdaneta M, Liu Y, Christopherson M, Prakash S, Abshire P and Smela E 2005 Integrating conjugated polymer microactuators with CMOS sensing circuitry for studying living cell Proc. Smart Structures and Materials, Electroactive Polymer Actuators and Devices (EAPAD) (San Diego, CA)

[16] Smela E 2003 Conjugated polymer actuators for biomedical applications Adv. Mater. 15 481-94

[17] Pettersson P F, Jager E W H and Inganas O 2000 Surface micromachined polymer actuators as valves in PDMS microfuidic system 1st Annual Int. IEEE-EMBS Special Topic Conf. on Microtechnologies in Medicine and Biology, (Lyon, France, 12-14 Oct.) pp 334-5

[18] Ebefors T, Mattsson J U, Kalvesten E and Stemme G 1999 A walking silicon micro-robot Proc. 10th Int. Conf. Solid-State Sensors and Actuators (TRANSDUCERS99) (Sendai, Japan, 7-10 Jun.) pp 1202-5

[19] Beneche W and Riethmuller W 1989 Application of silicon-microactuators based on bimorph structures Proc. IEEE MEMS '89 (Salt Lake City, USA, 20-22 Feb.) pp 116-20

[20] Lee K B and Lin L W 2007 A surface-micromachined vertical scanning micromirror $J$ Micromech. Microeng. 17 1394-401

[21] Fang Y, Tan X and Alici G 2008 Robust adaptive control of conjugated polymer actuators IEEE Trans. Control Syst. Technol. 16 600-12

[22] John S W, Alici G and Cook C D 2008 Validation of a resonant frequency model for polypyrrole trilayer actuators IEEE/ASME Trans. Mechatronics 13 401-9

[23] Lambda Physics Laser http://cmi.epfl.ch/backend/lambda.php (accessed June 2008)

[24] Frisch-Fay R 1962 Flexible Bars (London: Butterworth) 